\title{
Forschungsförderung in Deutschland
}

\author{
Gesprächsleitung: Achim Rody \\ Teilnehmer: Franz Ameismeier ${ }^{b}$ Renate Haidinger ${ }^{c} \quad$ Andreas Schneeweiss $^{d}$ \\ ${ }^{a}$ Klinik für Gynäkologie und Geburtshilfe, J.W.-Goethe-Universität, Frankfurt/M., \\ ${ }^{\mathrm{b}}$ GlaxoSmithKline GmbH \& Co. KG, \\ ${ }^{\circ}$ Brustkrebs Deutschland e.V., München, \\ ${ }^{d}$ Universitäts-Frauenklinik und Nationales Centrum für Tumorerkrankungen, Heidelberg, Germany
}

\section{Frage 1: Wie bewerten Sie die Zukunftsperspektive des wissenschaftlichen Nachwuchses?}

Ameismeier: Bezogen auf die berufliche Perspektive gut. Bezogen auf den wissenschaftlichen Schwerpunkt mit translationaler Ausrichtung allerdings schlechter als bei Internistischen Onkologen. Den Grund sehe ich darin, dass in der Gynäkologie die Zukunftsperspektive primär über die OP-Fertigkeiten, die Geburtshilfe und die angewandte Tumortherapie definiert wird.

Haidinger: Schlecht, es wird zu wenig dafür getan und es gibt zu wenig Anerkennung.

Schneeweiss: Schlecht, da der wissenschaftliche Nachwuchs in Deutschland zerrieben wird zwischen klinischem Alltag und Wissenschaft am Wochenende oder in der Freizeit. Die Strukturen müssten reformiert und ein Curriculum eingeführt werden, das nach amerikanischen Vorbild klinische und wissenschaftliche Ausbildung verzahnt und nicht «chaotisch» nebeneinander laufen lässt.

Frage 2: Halten Sie das derzeitige Begutachtungssystem wissenschaftlicher Anträge für adäquat? Welche Optimierungsmöglichkeiten halten Sie für vordringlich?

Ameismeier: Für die onkologischen Projekte insgesamt gesehen ist es ok. Wenn Sie die gynäkologischen Projekte unterrepräsentiert sehen, dann kann es an einer geringen Zahl eingereichter Anträge oder aber an der Qualität liegen. Beides kann ich nicht beurteilen.
Haidinger: Der Antragsteller muss schneller eine Nachricht erhalten, ob er überhaupt in der engeren Wahl ist, damit er ggf. auch woanders einen Antrag stellen kann.

Schneeweiss: Das Begutachtungssystem ist antiquiert und nicht mehr den aktuellen Erfordernissen angemessen. Es ist zu langsam, zu kompliziert und zu wenig mutig.

Frage 3: «Hochwertige Forschung schließt hochwertige klinische Ausbildung im Wesentlichen aus" - geht beides? Wenn ja, unter welchen Voraussetzungen ?

Ameismeier: Ich würde nicht behaupten, dass sich beides gegenseitig ausschließt. Es gibt ja motivierte junge Gynäkologen. Die Frage ist: Gibt es für die «Forscher» nach deren Facharztausbildung und Habilitation adäquate Stellen, die die Forschung und therapeutische Anwendung als Schwerpunkt haben und die weiteren Bereiche wie Geburtshilfe, etc. ausklammern?

Haidinger: Die jungen Ärzte müssen einen Teil der Zeit freigestellt werden, damit es nicht nur Forschung in der Freizeit gibt. Hierfür müssen Gelder bereitgestellt werden.

Schneeweiss: Es geht beides, wenn es aufeinander abgestimmt in Form eines straff organisierten Curriculums erfolgt.

\section{Frage 4: «Keine Zeit, keine Lust?» - wie kann das Interesse an wissenschaftlichen Fragestellungen bei Klinikern gesteigert werden?}

Ameismeier: Hier könnte eine Verbesserung der Berufsperspektive einen positiven Beitrag leisten.

PD Dr. med. Achim Rody, Klinik für Gynäkologie und Geburtshilfe

Johann-Wolfgang-Goethe-Universität

Theodor-Stern-Kai 7, 60590 Frankfurt/M., Germany

Tel. +49-69-6301-4117

Fax +49-69-6301-83469

achim.rody@em.uni-frankfurt.de 
Haidinger: Die jungen Ärzte brauchen mehr Zeit, um nicht nur auf Kosten des Privatlebens forschen zu können. Politik und Kliniken sind hier gefordert, ein neues Modell zu entwickeln.

Schneeweiss: Gebt den jungen Wissenschaftlern eine bessere Anleitung durch Einbindung in gute Arbeitsgruppen, dann werden Sie wieder «Lust» auf Forschung entwickeln und fruchtbar arbeiten.

\section{Frage 5: "Ich habe eine Idee, aber kein Geld!» - Wie sieht der optimale wissenschaftliche Start aus, was würden Sie jungen Kollegen empfehlen?}

Haidinger: Es müsste Aufstellungen geben, in denen genau definiert wird, wo, wofür und unter welchen Umständen es Forschungsgelder gibt.

Schneeweiss: Suche dir eine gut organisierte, seit Jahren erfolgreich publizierende Arbeitsgruppe mit etablierten Methoden, die als enger Kooperationsverbund aus klinischen Wissenschaftlern und Grandlagenforschern relevante Probleme/ Fragestellungen erforscht.

\section{Frage 6: Frau, Forschung, Habilitation und Familie - die Quadratur des Kreises oder was muss sich ändern?}

Ameismeier: Ich glaube dies ist zum Großteil eine persönliche Entscheidung, die die Familie treffen muss. Ohne Abstriche wird es nicht gehen.
Haidinger: Die berufstätige Frau muss besser gefördert werden: bessere, gezieltere Förderung, sofern die Frauen in die Forschung wollen oder auch Forschung betreiben wollen, es muss Versorgungsangebote für Kinder berufstätiger Frauen geben. Spezielle Programme für Frauen könnten von den Kliniken bzw. durch die Politik oder Krebshilfe gefördert werden.

Schneeweiss: Frau Prof. Nadia Harbeck fragen!

\section{Teilnehmer}

Dr. Franz Ameismeier

Head of Marketing \& Sales Hospital/Oncology

GlaxoSmithKline $\mathrm{GmbH} \& \mathrm{Co} . \mathrm{KG}$

Theresienhöhe 11

80339 München, Germany

Tel. +49 89 36044-8450, Fax -98450

franz.ameismeier@gsk.com

Renate Haidinger

1. Vorsitzende

Brustkrebs Deutschland e.V.

Charles-de-Gaulle-Str. 6

81737 München, Germany

Tel. +49 89 4161-98 00, Fax -98 01

renate.haidinger@brustkrebsdeutschland.de

Prof. Dr. med. Andreas Schneeweiss

Sektionsleiter Gynäkologische Onkologie

Universitäts-Frauenklinik und Nationales Centrum für

Tumorerkrankungen

Voss-Strasse 9

69115 Heidelberg, Germany

Tel. +49 6221 56-7856, Fax -7920

andreas.schneeweiss@med.uni-heidelberg.de 